\title{
Electric Vehicle Charging Station Location Decision Analysis for a Two-Stage Optimization Model Based on Shapley Function
}

\author{
Lifeng Yang $\mathbb{D}^{1},{ }^{1}$ Zhongwei Cheng $\mathbb{D},{ }^{1}$ Baojie Zhang $\mathbb{D},{ }^{2}$ and Fengyun $\mathrm{Ma}^{3}$ \\ ${ }^{1}$ School of Economics, Fuyang Normal University, Fuyang 236037, China \\ ${ }^{2}$ School of Business, Fuyang Normal University, Fuyang 236037, China \\ ${ }^{3}$ Hebei North Diesel Engine Co., Ltd., Zhongshan West Road 198, Shijiazhuang 050081, China \\ Correspondence should be addressed to Zhongwei Cheng; 2019111061@stu.fynu.edu.cn and Baojie Zhang; 19210501@ \\ stu.fynu.edu.cn
}

Received 30 May 2021; Accepted 23 July 2021; Published 2 August 2021

Academic Editor: Kenan Yildirim

Copyright (C) 2021 Lifeng Yang et al. This is an open access article distributed under the Creative Commons Attribution License, which permits unrestricted use, distribution, and reproduction in any medium, provided the original work is properly cited.

\begin{abstract}
The promotion of electric vehicles and their charging facilities to achieve carbon emission reduction is a research hotspot in the field of transportation. Aiming at the comprehensive decision of electric vehicle charging station (EVCS) location, this paper constructs an EVCS location evaluation index system that includes five indexes of grid load, traffic facilities, user preference, construction cost, and service radius. Firstly, we convert the exact number into interval judgment matrix, introduce Shapley fuzzy measure to calculate the weight of factors, and use the two-stage optimization model to further optimize the weight. Then, we combine the multiple criteria decision-making (MCDM) method in the Pythagorean fuzzy environment with partitioned normalized weighted Bonferroni mean (PFPNWBM) operator, and calculate the optimal ranking of alternatives according to the performance function and the accuracy function. Finally, a numerical example is used to analyze the difference between first-order linear optimization and two-stage optimization in alternative scheme evaluation, and the practical value of using model to evaluate EVCS location is verified.
\end{abstract}

\section{Introduction}

Under the pressure of carbon emission reduction and carbon neutrality, the promotion and use of new energy vehicles represented by electric vehicles are becoming increasingly popular. The location and construction of EVCS have attracted extensive attention in the academic community [1]. EVCS location is affected by many objective and subjective factors such as service radius [2], electric vehicle (EV) mileage [3], and user preference [4]. There is great uncertainty in the decision-making process [5], and it becomes increasingly impossible to assign accurate values in the evaluation scheme [6]. Fuzzy set theory [7] and MCDM model [8], which rely on subjective judgment data selection, are often used in the scheme selection of such problems [9].

The evaluation form of ordinary fuzzy sets cannot adequately describe the actual situation related to a large number of fuzziness and uncertainties [10]. To cope with this challenge, Zeng et al. [11] proposed the concepts of weighted double hesitation fuzzy set (WDHFS) and weighted double hesitation fuzzy element (WDHFE) and applied them to group decision-making. Fan and Xiao [12] proposed a twodimensional intuitionistic fuzzy set (TDIFS) model to integrate the uncertainty and reliability expression of intuitionistic fuzzy set (IFS), which can carry more information than simple indexes. In order to accurately reflect the relative relationship between alternatives, Tao et al. [13] extended the classical alternative queuing method (AQM) by using fuzzy preference relation (FPR). However, since IFS can only describe the case where the sum of membership and nonmembership does not exceed 1 [14], Yager and Abbasov [15] successfully extended IFS to Pythagorean fuzzy set (PFS), which allows a larger space to describe certain, uncertain, and hesitant information. PFS method is used in the selection of electric bicycle sharing recycling suppliers [16], on-site evaluation of household waste processing plants [17], 
and other optimization scheme selection decisions. Due to the uncertainty of the reality in the Pythagorean fuzzy environment, the choice of weight is often restricted by objective constraints. Based on this, Nie et al. [18] defined the constraints of incomplete known weights and built a linear programming model with the maximum performance value of the alternatives schemes as the optimal goal. The weight acquisition process of Shapley fuzzy metric was optimized.

In addition, Wang et al. [19] developed a rapid charging facility planning model by considering battery deterioration and vehicle heterogeneity within the driving range. The corresponding MCDM conceptual framework is also widely used in location decisions. Ni et al. [20], in the framework of MCDM, proposed a comprehensive model to characterize vaccination decision-making process influenced by society. Gao et al. [21] suggested a MCDM method based on probabilistic language term set and regret theory. For the significance of Shapley fuzzy measure method in measuring the importance of elements and the interaction characteristics of combinations between elements, Tan et al. [22] used Shapley comprehensive authorization benefit distribution method to analyze the energy storage and charging pile project. And this method is often used in conjunction with MCDM to study program decision-making problems. Jing et al. [23] proposed a decision-making method of integrated product conceptual scheme based on Shapley value method and fuzzy logic model.

Yang and Ma [24] believed that, with the increase of the order of the judgment matrix, the granularity of the scale is not meticulous enough, which limits the consistency of the judgment matrix. There is a trade-off between simplicity and precision when choosing a digital scale. Pasman and Rogers [25] proposed that expert opinions are enveloped in uncertainty, which includes accidental uncertainty (lack of accuracy and precision observation means) and cognitive uncertainty (quantity and quality of data). Xu and Hua [26] found that, in view of the differences in professional knowledge, problem familiarity, social background, and other aspects of the evaluation team members, they are more inclined to represent the evaluation situation by their own preferred information forms, so that the evaluation results often present a complex situation where multiple types of information coexisted. Due to the difficulty and contradiction of subjective judgment, as well as the time pressure, incomplete relative information of factors in the decisionmaking process, limitations of experts' expertise, and other reasons [27], Teng and Liu [28] proposed a new large group decision method in probabilistic language to analyze the interaction between interest subgroups and the relationship between criteria. Li [29] believed that because different quantitative attributes have different physical dimensions, normalization processing is needed to transform the exact number into an interval fuzzy number with membership degree and nonmembership degree.

In Section 2, we briefly review the concepts of PFS and Shapley fuzzy measure on the basis of existing research results. In Section 3, based on the review of relevant research literature, a systematic and complete evaluation index system of EVCS is constructed, and then an empirical study is conducted on 5 regions with large charging demand. In Section 4, we establish a two-stage optimization model to identify the optimal Shapley fuzzy measure under the background that incomplete weight information is known, which is the basis for determining the standard weight. Then, based on the PFPNWBM operator, performance, and accuracy function, the comprehensive performance value of each alternative scheme is calculated, so as to determine the optimal scheme ranking. Section 5 provides detailed resolution steps. Section 6 illustrates the availability of the proposed method through an example. Finally, Section 7 compares the differences between the optimal Shapley fuzzy measure identified by the two-stage dynamic optimization model and the first-order linear optimization model through a numerical example. The research proves that the evaluation index system and the evaluation method have good practicability, accuracy, and flexibility.

In summary, from the perspective of EVCS location decision analysis, this paper constructs a comprehensive and systematic EVCS location evaluation index system that includes five indexes of grid load, traffic facilities, user preference, construction cost, and service radius. And the heterogeneity of experts and the incomplete information of the weights in the evaluation are well dealt with by establishing a two-stage optimization model to identify the optimal Shapley fuzzy measure, which provides some theoretical support and practical methods for EVCS location construction.

\section{Preliminaries}

In this section, some basic concepts and theoretical methods are introduced, which conclude IFS, PFS, and Shapley function.

\subsection{Intuitionistic Fuzzy Set}

Definition 1 (see [30]). Let $X$ be a finite universe of discourse. $A=\left\{\left\langle x, \mu_{A}(x), v_{A}(x) \mid x \in X\right\rangle\right\}$ is defined as an IFS with the condition:

$$
\begin{aligned}
& \mu_{A}(x), v_{A}(x): X \longrightarrow[0,1], \\
& 0 \leq \mu_{A}(x)+v_{A}(x) \leq 1 .
\end{aligned}
$$

The $\mu_{A}(x), v_{A}(x)$ is the membership degree of $x \in X$ indicating the degree of support and unsupported.

\subsection{Pythagorean Fuzzy Set}

Definition 2 (see [31]). Let $X$ be the domain of argument. $A=\left\{\left\langle x, A\left(\mu_{A}(x), v_{A}(x)\right)\right\rangle, \mid x \in X\right\}$ is a PFS on $X$, where $\mu_{A}(x), v_{A}(x): X \longrightarrow[0,1]$ represents the membership and nonmembership of element $X$ to $A$ and $0 \leq\left(\mu_{A}(x)\right)^{2}+\left(v_{A}(x)\right)^{2} \leq 1$, respectively. The degree of indeterminacy $\pi_{A}(x)$ is expressed by $\pi_{A}(x)$ $=\sqrt{1-\left(\mu_{A}(x)\right)^{2}-\left(v_{A}(x)\right)^{2}}$. 
Definition 3 (see [32]). Let $a=A\left(\mu_{a}, v_{a}\right)$ be a PFN, and its score function and accuracy function are defined as follows:

$$
\begin{aligned}
S(a) & =\mu_{a}^{2}-v_{a}^{2}, \\
H(a) & =\mu_{a}^{2}+v_{a}^{2}, \quad \text { where } S(a), H(a) \in[0,1] .
\end{aligned}
$$

Definition 4 (see [33]). Let $a_{1}=A\left(\mu_{a_{1}}, v_{a_{1}}\right)$ and $a_{2}=A\left(\mu_{a_{2}}, v_{a_{2}}\right)$ be two PFNs. Then, the distance between $a_{1}$ and $a_{2}$ is expressed as

$$
d\left(a_{1}, a_{2}\right)=\sqrt{\frac{1}{2}\left[\left(\left(\mu_{a_{1}}\right)^{2}-\left(\mu_{a_{2}}\right)^{2}\right)^{2}+\left(\left(v_{a_{1}}\right)^{2}-\left(v_{a_{2}}\right)^{2}\right)^{2}+\left(\left(\pi_{a_{1}}\right)^{2}-\left(\pi_{a_{2}}\right)^{2}\right)^{2}\right]} .
$$

As shown in Figure 1, the applicable scope of PFS and IFS is $1 / 4$ circle and right triangle in the first quadrant of the plane Cartesian coordinate system, respectively. Obviously, the use range of PFS is larger than that of IFS, so PFS has a wider computing space.

\subsection{Shapley Function}

Definition 5 (see [34]). Let $X$ be a finite set, and $P(x)$ is the power set of $X$. The fuzzy measure $\mu$ defined on $X: P(x) \longrightarrow[0,1]$ satisfies the following conditions:
(1) $\mu(\Phi)=0, \mu(X)=1$

(2) If $A, B \in P(x)$ and $A \subseteq B$, then $\mu(A) \subseteq \mu(B)$

Shapley function is used to measure the income distribution of each member alliance in the game. With respect to the fuzzy measure $\mu(A)$ on the finite set defined as above, it can be expressed as

$$
\varphi_{i}(\mu, N)=\mu(i)=\sum_{S \subseteq N / i} \frac{(n-s-1) ! s !}{n !}(\mu(S \cup i)-\mu(i)), \quad \forall i \in N .
$$

\section{The Evaluation Index System of EVCS Location}

With the rapid development of EV technology, EV has become a major participant in the future development of new energy vehicles. However, due to the limited number of fast chargers and the increase of residents' charging demands, EV charging operation may encounter the problems of increased queuing delay and increased charging operation costs [35]. To this end, governments around the world are promoting the use of EV in possible ways and issuing various preferential policies to encourage EV enterprises to cooperate with energy companies to build EVCS [4]. Therefore, establishing a comprehensive and complete EVCS location evaluation index system is the most effective way to promote the popularization of $\mathrm{EV}$ and solve the contradiction between supply and demand. Based on relevant literature review and analysis, we select the following five indicators from 10 indicators, such as grid load, traffic facilities, user preference, construction cost, service radius, EV ownership, driving mileage, policy support, regional service level, and elastic demand [2-4, 35-37].

3.1. Grid Load. Strong grid load capacity is the basic requirement of EVCS construction. Grid load refers to the ability of regional grid to withstand voltage load. The simultaneous charging of many vehicles in a fixed station by quick charging technology may produce huge additional demands on the power grid and may lead to power loss, which is bound to have certain requirements on the bearing capacity and supply level of grid.

3.2. Traffic Facilities. The location of EVCS directly affects the cost of driving on the road for vehicle owners (time and energy consumption on the way to the charging station). Electric vehicle users will give priority to EVCS with short travel distance and convenient road passage, so as to reduce driving costs. Therefore, the perfection of regional traffic facilities, namely, the minimization of customer travel costs is an important factor affecting EVCS location.

3.3. User Preference. User preference is mainly for users themselves, that is, drivers may form the habit of charging battery electric vehicle (BEV) after daily travel, including the battery capacity of the purchased electric vehicles, the acceptable charging time, the familiarity with public charging stations and travel mode (total travel duration of the next travel day, day interval between the start of the next travel and the start of the current charging event), etc.

3.4. Construction Cost. EVCS location should minimize the total cost and network loss cost of the charging station during the planning period as the goal of the optimal planning of the charging station [38]. The construction cost of charging station 


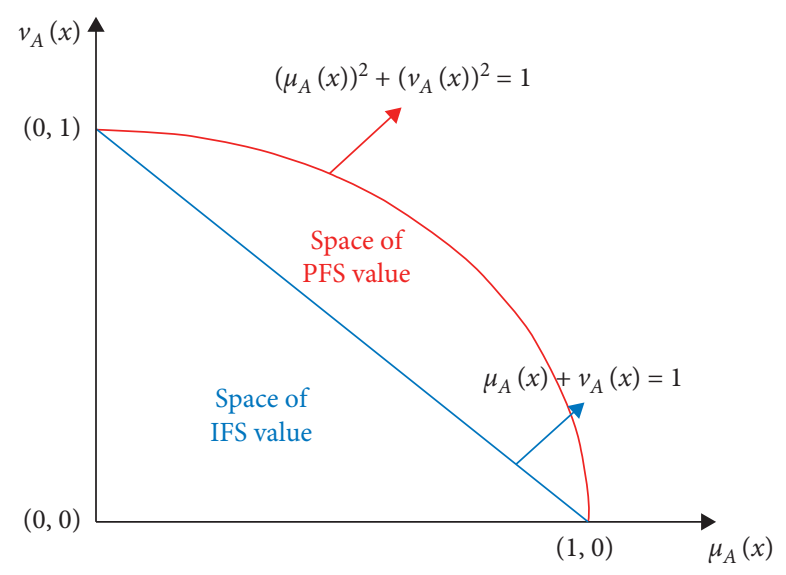

FIGURE 1: IFS and PFS applicable scope comparison.

location includes investment cost, operating cost, and maintenance cost. Among them, investment cost refers to the investment cost per unit capacity of transformers and charging equipment, investment cost per unit capacity of other equipment except transformers and charging equipment, and land use cost. Operating cost includes charging cost, electric energy consumption cost of electrical equipment, filtering compensation cost of charging station, and labor cost. Maintenance cost refers to the maintenance cost per unit capacity of transformers and charging equipment and the maintenance cost per unit capacity of other equipment except transformers and charging equipment.

3.5. Service Radius. When the number of charging station is large, the smaller the Voronoi diagram area of the service area of the corresponding charging stations is, the lower the driving cost of the vehicle owner will be. In order to maximize EV battery cycle life and EVCS resources, on the premise of satisfying users' charging needs, the service radius of charging station should be less than the actual distance between two adjacent charging stations under the premise of meeting the charging needs of the user and at most equal to the reasonable continuous driving distance of electric vehicle at a constant speed.

\section{EVCS Location Evaluation Method}

4.1. Conversion of Exact Numbers. Comparison scale refers to the pairwise comparison of the relative importance of the indicators and gives the exact number judgment result according to one's own preference. We use the 1-9 scale: the range of $a_{i j}$ is going to be in the range $1,2, \ldots, 9$. When making qualitative pairwise comparisons, people usually have five distinct levels in mind. Assume that $m$ schemes comprise scheme set $X=\left\{x_{1}, x_{2}, \ldots, x_{m}\right\}$ and $n$ evaluation indicators are divided into index set $C=\left\{c_{1}, c_{2}, \ldots, c_{n}\right\}$, and then the exact scoring value of the $j$ evaluation object given by experts on the $i$ indicator performance is $a_{i j}$. It can be transformed into an intuitionistic fuzzy set by the following formula:

$$
\begin{aligned}
& \mu_{i j}= \begin{cases}\alpha_{i} \frac{a_{i j}}{a_{i}^{\max }}, & i \in \Omega_{b}, \\
\delta_{i} \frac{a_{i}^{\min }}{a_{i j}}, & i \in \Omega_{c}, a_{i}^{\min } \neq 0, \\
\delta_{i}\left(1-\frac{a_{i j}}{a_{i}^{\max }}\right) & i \in \Omega_{c}, a_{i}^{\min }=0,\end{cases} \\
& v_{i j}= \begin{cases}\beta_{i} \frac{a_{i j}}{a_{i}^{\max }}, & i \in \Omega_{b}, \\
\gamma_{i} \frac{a_{i}^{\min }}{a_{i j}}, & i \in \Omega_{c}, a_{i}^{\min } \neq 0, \\
\gamma_{i}\left(1-\frac{a_{i j}}{a_{i}^{\max }}\right), & i \in \Omega_{c}, a_{i}^{\min }=0,\end{cases}
\end{aligned}
$$

where $\Omega_{b}$ and $\Omega_{c}$ represent the set of cost-type and benefittype indicators, respectively. The determination method of $a_{i}^{\min }$ and $a_{i}^{\max }$ is as follows:

$$
\begin{aligned}
& a_{i}^{\min }=\min _{1 \leq j \leq n}\left\{a_{i j}\right\}, \\
& a_{i}^{\max }=\min _{1 \leq j \leq n}\left\{a_{i j}\right\} .
\end{aligned}
$$

$\alpha_{i}, \beta_{i}, \gamma_{i}$, and $\delta_{i}$ are four adjustment parameters, which are given by the actual decision-making environment and decision-maker's experience [29]. Their values are all between 0 and 1 , and they meet $0 \leq \alpha_{i}+\beta_{i j} \leq 1$ and $0 \leq \alpha_{i}+\beta_{i} \leq 1$. Accordingly, the exact number ${ }_{a}^{i j}$ can be transformed into an interval fuzzy number $A_{i j}=\left(\mu_{i j}, v_{i j}\right)$.

4.2. Identifying the Optimal Shapley Fuzzy Measure. In practical MCDM issues, the weights of the attributes and decision makers (DM) are always partially known or completely unknown. To overcome this limitation, Wang et al. [39] developed the maximized deviation method 
(MDM) to handle more complex decision applications. It is well known that it is easier to choose better alternatives by ranking the fusion value, so as to more clearly reflect the differences between all alternatives. The main principle of MDM is that when an attribute has a significant impact on the ranking priority results, it is obvious that the attribute should be given greater weight in influencing the decision results. Conversely, if an attribute causes only a small change in the order of alternatives, it means that the attribute has less impact on the decision process and should be given less weight.

Set the scheme set $X=\left\{x_{1}, x_{2}, \ldots, x_{m}\right\}, n$ evaluation index set $C=\left\{c_{1}, c_{2}, \ldots, c_{n}\right\}$, and weighted vector set $w=\left\{w_{1}, w_{2}, \ldots, w_{n}\right\}$. Under the same criteria, the set of deviation values of the pairwise alternatives is $d\left(a_{i j}, a_{k j}\right)$. Then we can calculate the degree of difference between each alternative through MDM. The calculation formula of deviation is as follows:

$$
\begin{aligned}
& D_{j}(\varphi)=\sum_{i=1}^{m} D_{i j}(\varphi)=\sum_{i=1}^{m} \sum_{k=1}^{m} d\left(a_{i j}, a_{k j}\right) \varphi_{j}\left(\mu, P_{h}\right), \\
& j=1,2, \ldots, n .
\end{aligned}
$$

In order to determine the optimal Shapley fuzzy measure, this paper establishes a two-stage dynamic programming model, which aims to achieve the maximum deviation of all standards.

$$
\begin{aligned}
& \max D(\varphi)=\sum_{j=1}^{n} \sum_{i=1}^{m} \sum_{k=1}^{m} d\left(a_{i j}, a_{k j}\right) \varphi_{j}\left(\mu, P_{h}\right) \\
& \text { s.t. }\left\{\begin{array}{l}
\mu\left(P_{h}\right)=1, \\
\mu(S) \leq \mu(T), \quad \forall S, T \subseteq P_{h}, S \subseteq T, \\
\mu(j) \in U_{j}, \quad \forall j \in P_{h} .
\end{array}\right.
\end{aligned}
$$

4.3. Calculating the Comprehensive Performance Value. Let $r, q \geq 0, a_{i}=A\left(\mu_{a i}, v_{a i}\right)(i=1,2, \ldots, m)$ be a collection of PFNs, the aggregated results derived by PFPNWBM [18] operator are still a PFN, and it is expressed as

$$
\begin{aligned}
& \operatorname{PFPNWBM}\left(a_{1}, a_{2}, \ldots, a_{m}\right)=\left(\sqrt{1-\left(\prod_{h=1}^{d}\left(1-\left(1-\left(1-\mu_{a_{i}}^{2 r} \mu_{a_{j}}^{2 q}\right)^{\left(\varphi_{i, h}\left(\mu, P_{h}\right) \varphi_{j, h}\left(\mu, P_{h}\right) /\left(1-\varphi_{i, h}\left(\mu, P_{h}\right)\right)\right)}\right)^{(1 /(r+q))}\right)\right)^{(1 / d)}},\right. \\
& \prod_{h=1}^{d}\left(\sqrt{\left.\left.1-\left(1-\prod_{\substack{i, j \in P_{h} \\
i \neq j}}\left(1-\left(1-v_{a_{i}}^{2}\right)^{r}\left(1-v_{a_{j}}^{2}\right)\right)^{\left(\varphi_{i, h}\left(\mu, P_{h}\right) \varphi_{j, h}\left(\mu, P_{h}\right) /\left(1-\varphi_{i, h}\left(\mu, P_{h}\right)\right)\right)}\right)^{(1 /(r+q))}\right)^{(1 / d)}\right) .}\right.
\end{aligned}
$$

\section{The Decision Steps and the Characteristics}

In this section, the detailed steps of the evaluation index system of EVCS location are provided:

Step 1: establish a normalized judgment matrix.

We adopt a 1-9 comparative scale and invited experts to give an exact score of $a_{i j}$ for the performance of the $J_{\text {th }}$ evaluation object under the $I_{\text {th }}$ indicator according to their preferences. If there are cost criteria, the decision matrix $A=\left(a_{i j}\right)_{m \times n}$ need to be normalized by the following equations [18]:

$$
\widetilde{a_{i j}}= \begin{cases}a_{i j}, & \text { for benefit criteria, } \\ \left(a_{i j}\right)^{c}=\left(v_{a}, u_{a}\right), & \text { for cost criteria. }\end{cases}
$$

Step 2: calculate the distance of each alternative.

The distance between the alternative scheme $I$ and $k$ under the standard $j$ can be calculated by the formula. Step 3: determine the optimal Shapley fuzzy measure.
Using the above distance value and formula (7), the linear programming model is solved and the optimal Shapley fuzzy measure is obtained.

Step 4: use formula (9) to calculate the comprehensive performance value of each alternative.

Step 5: use formula (2) to calculate the value of score and accuracy function.

The decision frame is shown in Figure 2.

\section{An Illustrative Example}

To test the practicality of the EVCS location evaluation index system, we select five optional areas $X=\left\{x_{1}, x_{2}, x_{3}, x_{4}, x_{5}\right\}$ : electric refueling area $x_{1}$, high-speed service area $x_{2}$, user residential area $x_{3}$, commercial shopping area $x_{4}$, and civic work area $x_{5}$. The index set consists of five evaluation indicators: grid load $C_{1}$, traffic facilities $C_{2}$, user preference $C_{3}$, construction cost $C_{4}$, and service radius $C_{5}$. Based on the correlation pattern, it is assumed that the criteria are divided into two categories $P_{1}=\left\{c_{1}, c_{2}, c_{3}\right\}$ and $P_{2}=\left\{c_{4}, c_{5}\right\}$. The above potential alternatives are evaluated by experts 


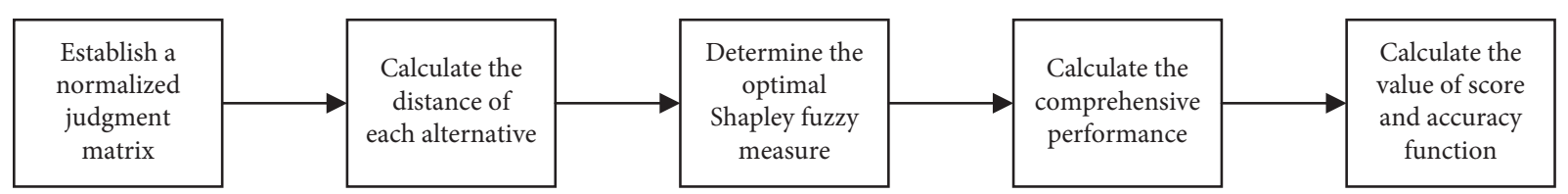

Figure 2: EVCS site selection decision diagram.

according to these criteria using Pythagorean fuzzy information. This assessment provides incomplete information on importance weights:

$$
\begin{aligned}
& U_{1}=[0.25,0.4], \\
& U_{2}=[0.4,0.5], \\
& U_{3}=[0.35,0.4], \\
& U_{4}=[0.45,0.6], \\
& U_{5}=[0.3,0.5] .
\end{aligned}
$$

Now the method in the paper can be used to solve the problem. The detailed decision steps are shown as follows :

Step 1: establish the normalized judgment matrix.

The exact quantitative evaluation matrix given by experts is shown in Table 1, which is converted into Table 2 by formula (10). As the cost types, they should be normalized into Table 3 by formula (5).

Step 2: calculate the distance of the alternative.

Under the same criteria, the distance values of pairwise schemes obtained by using the formula (3) are shown in Table 4.

Step 3: determine the optimal Shapley fuzzy measure. The indexes are divided into $p_{1}$ and $p_{2}$, and their

\begin{tabular}{|c|c|c|c|c|c|}
\hline & $c_{1}$ & $c_{2}$ & $c_{3}$ & $c_{4}$ & $c_{5}$ \\
\hline$x_{1}$ & $(0.26,0.17)$ & $(0.20,0.13)$ & $(0.12,0.80)$ & $\begin{array}{c}(0.43 \\
0.29)\end{array}$ & $\begin{array}{c}(0.60, \\
0.40)\end{array}$ \\
\hline$x_{2}$ & $\begin{array}{l}(0.33 \\
0.22)\end{array}$ & $\begin{array}{l}(0.33 \\
0.22)\end{array}$ & $\begin{array}{l}(0.60 \\
0.40)\end{array}$ & $\begin{array}{l}(0.33 \\
0.22)\end{array}$ & $\begin{array}{l}(0.43, \\
0.29)\end{array}$ \\
\hline$x_{3}$ & $(0.20,0.13)$ & $(0.20,0.13)$ & $\begin{array}{l}(0.30 \\
0.20)\end{array}$ & $\begin{array}{c}(0.60 \\
0.40)\end{array}$ & $\begin{array}{l}(0.60, \\
0.40)\end{array}$ \\
\hline$x_{4}$ & $(0.12,0.08)$ & $(0.12,0.08)$ & $(0.12,0.08)$ & $\begin{array}{c}(0.60 \\
0.40)\end{array}$ & $\begin{array}{c}(0.30 \\
0.20)\end{array}$ \\
\hline$x_{5}$ & $(0.12,0.08)$ & $(0.12,0.08)$ & $\begin{array}{c}(0.60 \\
0.40)\end{array}$ & $\begin{array}{c}(0.60 \\
0.40)\end{array}$ & $\begin{array}{l}(0.60, \\
0.40)\end{array}$ \\
\hline
\end{tabular}
Shapley fuzzy measure is calculated, respectively.
TABLE 1: Accurate number rating.

\begin{tabular}{lccccc}
\hline & $c_{1}$ & $c_{2}$ & $c_{3}$ & $c_{4}$ & $c_{5}$ \\
\hline$x_{1}$ & 8 & 4 & 3 & 6 & 7 \\
$x_{2}$ & 4 & 5 & 6 & 2 & 7 \\
$x_{3}$ & 3 & 4 & 2 & 6 & 5 \\
$x_{4}$ & 7 & 7 & 8 & 9 & 9 \\
$x_{5}$ & 2 & 1 & 4 & 3 & 5 \\
\hline
\end{tabular}

TABLe 2: Interval fuzzy number rating.

Taking the criteria in the first category $p_{1}$ as an example, Shapley fuzzy measure of each criterion can be obtained from equation (4)

$$
\begin{aligned}
& \varphi_{1}\left(\mu, P_{h}\right)=\frac{1}{3} \mu\left(c_{1}\right)+\frac{1}{6}\left(\mu\left(c_{1}, c_{2}\right)-\mu\left(c_{2}\right)+\mu\left(c_{1}, c_{3}\right)-\mu\left(c_{3}\right)\right)+\frac{1}{3}\left(1-\left(\mu\left(c_{2}, c_{3}\right)\right)\right), \\
& \varphi_{2}\left(\mu, P_{h}\right)=\frac{1}{3} \mu\left(c_{2}\right)+\frac{1}{6}\left(\mu\left(c_{1}, c_{2}\right)-\mu\left(c_{1}\right)+\mu\left(c_{2}, c_{3}\right)-\mu\left(c_{3}\right)\right)+\frac{1}{3}\left(1-\left(\mu\left(c_{1}, c_{3}\right)\right)\right), \\
& \varphi_{3}\left(\mu, P_{h}\right)=\frac{1}{3} \mu\left(c_{3}\right)+\frac{1}{6}\left(\mu\left(c_{1}, c_{3}\right)-\mu\left(c_{1}\right)+\mu\left(c_{2}, c_{3}\right)-\mu\left(c_{2}\right)\right)+\frac{1}{3}\left(1-\left(\mu\left(c_{1}, c_{2}\right)\right)\right) .
\end{aligned}
$$

Based on the above derivation, a linear programming model can be established as follows:

$$
\begin{aligned}
& \max \left[-0.4165 \mu\left(c_{1}\right)-0.0775 \mu\left(c_{2}\right)+0.4939 \mu\left(c_{3}\right)-0.4939 \mu\left(c_{1}, c_{2}\right)+0.0775 \mu\left(c_{1}, c_{3}\right)+0.4165 \mu\left(c_{2}, c_{3}\right)+1.4758\right] \\
& \text { s.t. }\left\{\begin{array}{l}
\mu(1,2,3)=1, \\
\mu(1) \leq \mu(1,2), \mu(1) \leq \mu(1,2), \mu(1) \leq \mu(1,3), \\
\mu(2) \leq \mu(1,3), \mu(2) \leq \mu(2,3), \mu(3) \leq \mu(2,3), \\
\mu(1) \in[0.25,0.4], \mu(2) \in[0.4,0.5], \mu(3) \in[0.35,0.4] .
\end{array}\right.
\end{aligned}
$$


TABLE 3: Normalized rating.

\begin{tabular}{lccccc}
\hline & $c_{1}$ & $c_{2}$ & $c_{3}$ & $c_{4}$ & $c_{5}$ \\
\hline$x_{1}$ & $(0.17,0.26)$ & $(0.40,0.60)$ & $(0.12,0.80)$ & $(0.29,0.43)$ & $(0.60,0.40)$ \\
$x_{2}$ & $(0.22,0.33)$ & $(0.40,0.60)$ & $(0.60,0.40)$ & $(0.22,0.33)$ & $(0.43,0.29)$ \\
$x_{3}$ & $(0.13,0.20)$ & $(0.27,0.41)$ & $(0.30,0.20)$ & $(0.40,0.60)$ & $(0.60,0.40)$ \\
$x_{4}$ & $(0.08,0.12)$ & $(0.32,0.48)$ & $(0.12,0.08)$ & $(0.40,0.60)$ & $(0.30,0.20)$ \\
$x_{5}$ & $(0.08,0.12)$ & $(0.40,0.60)$ & $(0.60,0.40)$ & $(0.40,0.60)$ & $(0.60,0.40)$ \\
\hline
\end{tabular}

TABLE 4: The same standard pairwise alternative distance.

\begin{tabular}{|c|c|c|c|c|c|}
\hline & $c_{1}$ & $c_{2}$ & $c_{3}$ & $c_{4}$ & $c_{5}$ \\
\hline$d\left(a_{1 j}, a_{2 j}\right)$ & 0.0723 & 0.0000 & 0.4429 & 0.1181 & 0.2230 \\
\hline$d\left(a_{1 j}, a_{3 j}\right)$ & 0.0352 & 0.2472 & 0.0969 & 0.2230 & 0.0000 \\
\hline$d\left(a_{1 j}, a_{4 j}\right)$ & 0.0673 & 0.1661 & 0.4429 & 0.2230 & 0.3460 \\
\hline$d\left(a_{1 j}, a_{5 j}\right)$ & 0.0673 & 0.0000 & 0.4429 & 0.2230 & 0.0000 \\
\hline$d\left(a_{2 j}, a_{3 j}\right)$ & 0.0965 & 0.2472 & 0.3460 & 0.3218 & 0.2230 \\
\hline$d\left(a_{2 j}, a_{4 j}\right)$ & 0.1231 & 0.1661 & 0.0000 & 0.3218 & 0.1230 \\
\hline$d\left(a_{2 j}, a_{5 j}\right)$ & 0.1231 & 0.0000 & 0.0000 & 0.3218 & 0.2230 \\
\hline$d\left(a_{3 j}, a_{4 j}\right)$ & 0.0322 & 0.0811 & 0.3460 & 0.0000 & 0.3460 \\
\hline$d\left(a_{3 j}, a_{5 j}\right)$ & 0.0322 & 0.2472 & 0.3460 & 0.0000 & 0.0000 \\
\hline$d\left(a_{4 j}, a_{5 j}\right)$ & 0.0000 & 0.1661 & 0.0000 & 0.0000 & 0.3460 \\
\hline
\end{tabular}

By solving the model, we can obtain

$$
\begin{aligned}
\mu(1) & =0.25, \\
\mu(2) & =0.4, \\
\mu(3) & =0.35, \\
\mu(1,2) & =0.5, \\
\mu(1,3) & =\mu(2,3)=1 .
\end{aligned}
$$

After a round of anonymous ratings by experts, the proceeds of the collaboration are returned to all parties. $\mu(1)+\mu(2) \geq \mu(1,2)$ is found, which means that the profit value after the two cooperation is lower than the value created separately. So they will choose the final cooperation. In order to guarantee the firmness and reliability of alliance cooperation, the benefits of all parties after cooperation should be at least greater than or equal to the sum of the benefits of all parties without cooperation. Therefore, on the basis of the original linear programming, we add constraint conditions:

$$
\mu\left(S_{m}\right)+\mu\left(S_{n}\right) \leq \mu(T), \quad \forall S_{m}, S_{n}, T \subseteq P_{h}, S_{m}, S_{n} \subseteq T .
$$

We can obtain $\mu(1)=0.25, \mu(2)=0.4, \mu(3)=$ $0.35, \mu(1,2)=1, \mu(1,3)=0.6$, and $\mu(2,3)=1$.

According to formula (4), the Shapley fuzzy measure is calculated as $\varphi_{1}\left(\mu, P_{h}\right)=0.2083, \varphi_{2}\left(\mu, P_{h}\right)=0.2833$, and $\varphi_{3}\left(\mu, P_{h}\right)=0.5084$.

Similar to the processing procedure of the criteria in $p_{1}$, for Shapley's fuzzy measure of $C_{3}$ and $C_{4}$ in $p_{2}$, we can get

$$
\begin{aligned}
& \varphi_{4}\left(\mu, P_{h}\right)=0.2, \\
& \varphi_{5}\left(\mu, P_{h}\right)=0.3 .
\end{aligned}
$$

Step 4: use formula (9) to calculate the comprehensive performance value of each alternative.

When $r=q=1$, the comprehensive performance value of each alternative can be calculated as

$$
\begin{aligned}
& a_{1}=[0.1609,0.4738], \\
& a_{2}=[0.1065,0.7398], \\
& a_{3}=[0.0354,0.6870], \\
& a_{4}=[0.1628,0.5836], \\
& a_{5}=[0.0941,0.7273] .
\end{aligned}
$$

Step 5: the score and accuracy function values are calculated using equation (2).

Performance function and accuracy function are shown in Table 5.

\section{Comparison and Analysis}

The application case shows that the constructed EVCS location evaluation system and evaluation method can prioritize the final alternatives and can better deal with problems such as the heterogeneity of experts and the incomplete information of the weights in the evaluation.

From the point of view of indicator weight, the importance of the five indicators of "grid load $C_{1}$, traffic facilities $C_{2}$, user preference $C_{3}$, construction cost $C_{4}$, and service radius $C_{5}$ " in EVCS location evaluation system is significantly different. The importance is ranked as follows: 
TABLE 5: Score and accuracy ranking.

\begin{tabular}{cccc}
\hline & $S\left(a_{i}\right)$ & $H\left(a_{i}\right)$ & Ranking \\
\hline$x_{1}$ & -0.1986 & 0.2504 & 2 \\
$x_{2}$ & -0.0566 & 0.2181 & 4 \\
$x_{3}$ & -0.6227 & 0.6273 & 1 \\
$x_{4}$ & -0.3141 & 0.3671 & 5 \\
$x_{5}$ & -0.4425 & 0.4750 & 3 \\
\hline
\end{tabular}

$C_{4} \prec C_{1} \prec C_{2} \prec C_{5} \prec C_{3}$. It can be seen that, for government EVCS location, understanding the charging preference and travel modes of regional users is the key to EVCS obtaining maximum profits. The service radius ranks second in importance, showing that regional charging demand is the basic requirement for EVCS to perform its service functions.

Judging from the evaluation results, the location of the 5 EVCS areas obtained by the two-stage optimization planning model is relatively good, especially the expressway, which has perfect infrastructure, convenient transportation, and huge passenger flow. Only through the first stage of decision-making, we get the comprehensive performance value of the five alternatives:

$$
\begin{aligned}
& a_{1}=[0.2705,0.8107], \\
& a_{2}=[0.1065,0.7398], \\
& a_{3}=[0.0354,0.6870], \\
& a_{4}=[0.1326,0.6541], \\
& a_{5}=[0.0941,0.7273] .
\end{aligned}
$$

The optimal ranking is $x_{3} \prec x_{5} \prec x_{4} \prec x_{1} \prec x_{2}$.

The results show that, due to the special complexity of the decision state, the two-stage optimization modifies the Shapley fuzzy measure, changes the original weight value, and then affects the optimal ranking of the final alternative. This supports the EVCS location evaluation method constructed by this research.

\section{Conclusions}

Using scientific methods to evaluate EVCS location is of great significance for improving the design, development, promotion, and application of EV. This paper builds a systematic EVCS location evaluation index system on the basis of comprehensive domestic and foreign research results on EV evaluation and various charging station location evaluation.

First of all, in order to better deal with the heterogeneity, fuzziness, and uncertainty of expert evaluation information, this study introduces 1-9 evaluation scale for qualitative paired comparison and constructs interval fuzzy quantitative evaluation matrix by converting the exact number into the intuitionistic fuzzy set and other relevant theoretical results. Moreover, a two-stage optimization model to identify the optimal Shapley fuzzy measure is established by fully considering the incomplete knowledge of weights in the actual decision-making environment. And five representative EVCS locations are taken as the research objects for empirical application. Finally, the practicability, effectiveness, and flexibility of the evaluation index system and evaluation method are verified by analyzing the difference of the evaluation results between the first-order linear optimization and the two-stage optimization.

Admittedly, this study also has some limitations, such as relatively small selection of research samples, insufficient geographical coverage, relatively small number of evaluators, and sample data collected. Although the heterogeneity of expert groups in the evaluation process has been considered, the diversity of evaluation criteria and the multigranularity of decision-making information have not been considered. In follow-up research, we will further expand research objects, enrich sample data, optimize research methods, and consider researching EVCS construction issues from the perspective of consumer experience, so as to enhance consumer experience and make it better to use charging services.

\section{Data Availability}

The data came from expert ratings.

\section{Conflicts of Interest}

The authors declare that they have no conflicts of interest.

\section{Acknowledgments}

This work was supported by Anhui Province Philosophy and Social Science Planning General Project (no. AHSKY2017D70).

\section{References}

[1] A. Pal, B. Aniruddha, and A. C. Kumar, "Allocation of electric vehicle charging station considering uncertainties," Sustainable Energy, Grids and Networks, vol. 25, Article ID 100422, 2021.

[2] Z. P. Liu, F. Wen, Y. S. Xue, and J. B. Xin, "The optimal location and determination of the electric vehicle charging station," Automation of Electric Systems, vol. 36, no. 3, pp. 54-59, 2012.

[3] S. Q. Zhao, Z. W. Li, and D. Lei, "Optimal location and capacity of electric vehicle charging station based on urban traffic network information," Electrical Automation Equipment, vol. 36, no. 10, pp. 8-15, 2016.

[4] M. Xu, Q. Meng, K. Liu, and T. Yamamoto, "Joint charging mode and location choice model for battery electric vehicle users," Transportation Research Part B: Methodological, vol. 103, pp. 68-86, 2017.

[5] Z. P. Chen and W. Yang, "An MAGDM based on constrained FAHP and FTOPSIS and its application to supplier selection," Mathematical and Computer Modelling, vol. 54, no. 11, pp. 2802-2815, 2011.

[6] A. Sotoudeh-Anvari, "A critical review on theoretical drawbacks and mathematical incorrect assumptions in fuzzy OR methods: review from 2010 to 2020," Applied Soft Computing, vol. 93, Article ID 106354, 2020.

[7] L. A. Zadeh, "Fuzzy sets," Information and Control, vol. 8, no. 3, pp. 338-353, 1965.

[8] C. Fu, M. Xue, W. Y. Liu, D. L. Xu, and J. B. Yang, "Datadriven preference learning in multiple criteria decision 
making in the evidential reasoning context," Applied Soft Computing, vol. 102, Article ID 107109, 2021.

[9] P. P. Ghaebi, M. Bornapour, R. Hemmati, and M. J. Guerrero, "Charging station stochastic programming for hydrogen/ battery electric buses using multi-criteria crow search algorithm," Renewable and Sustainable Energy Reviews, vol. 144, Article ID 111046, 2021.

[10] M. Krawczak and G. Szkatuła, "On matching of intuitionistic fuzzy sets," Information Sciences, vol. 517, pp. 254-274, 2020.

[11] W. Y. Zeng, Y. Xi, Q. Yin, and P. Guo, "Weighted dual hesitant fuzzy set and its application in group decision making," Neurocomputing, 2020.

[12] Y. Fan and F. Y. Xiao, "TDIFS: two dimensional intuitionistic fuzzy sets," Engineering Applications of Artificial Intelligence, vol. 95, Article ID 103882, 2020.

[13] R. Tao, Z. Liu, R. Cai, and K. H. Cheong, "A dynamic group MCDM model with intuitionistic fuzzy set: perspective of alternative queuing method," Information Sciences, vol. 555, pp. 85-103, 2021.

[14] W. F. Liu and H. Xia, "Pythagoras hesitates the fuzzy set," Fuzzy System and Mathematics, vol. 30, no. 4, pp. 107-115, 2016.

[15] R. R. Yager and A. M. Abbasov, "Pythagorean membership grades in multicriteria decision making," IEEE Transactions of Fuzzy Systems, vol. 22, no. 4, pp. 958-965, 2014.

[16] Y. Tang and Y. Yang, "Sustainable e-bike sharing recycling supplier selection: an interval-valued pythagorean fuzzy MAGDM method based on preference information technology," Journal of Cleaner Production, vol. 287, Article ID 125530, 2021.

[17] C. H. Zhang, Q. Q. Hu, S. Z. Zeng, and W. H. Su, "IOWLADbased MCDM model for the site assessment of a household waste processing plant under a pythagorean fuzzy environment," Environmental Impact Assessment Review, vol. 89, Article ID 106579, 2021.

[18] R. X. Nie, Z. P. Tian, J. Q. Wang, and J. H. Hu, "Pythagorean fuzzy multiple criteria decision analysis based on shapley fuzzy measures and partitioned normalized weighted bonferroni mean operator," International Journal of Intelligent Systems, vol. 34, no. 5, pp. 1-28, 2018.

[19] H. Wang, D. Zhao, Y. T. Cai, Q. Meng, and P. O. Ghim, “Taxi trajectory data based fast-charging facility planning for urban electric taxi systems," Applied Energy, vol. 286, Article ID 116515, 2021.

[20] L. Ni, Y.-W. Chen, and O. de Brujin, “Towards understanding socially influenced vaccination decision making: an integrated model of multiple criteria belief modelling and social network analysis," European Journal of Operational Research, vol. 293, no. 1, pp. 276-289, 2021.

[21] J. W. Gao, H. J. Men, F. J. Guo et al., "A multi-criteria decision-making framework for compressed air energy storage power site selection based on the probabilistic language term sets and regret theory," Journal of Energy Storage, vol. 37, Article ID 102473, 2021.

[22] Q. Tan, P. Wu, W. Tang et al., "Benefit allocation model of distributed photovoltaic power generation vehicle shed and energy storage charging pile based on integrated weightingshapley method," Global Energy Interconnection, vol. 3, no. 4, pp. 375-384, 2020.

[23] L. Jing, Y. Zhan, Q. Li et al., "An integrated product conceptual scheme decision approach based on shapley value method and fuzzy logic for economic-technical objectives trade-off under uncertainty," Computers \& Industrial Engineering, vol. 156, Article ID 107281, 2021.
[24] H. T. Yang and D. T. Ma, "Research on consistency of judgment matrix in analytic hierarchy process," Modern Electronic Technology, vol. 19, pp. 46-48, 2007.

[25] H. J. Pasman and W. J. Rogers, "How to treat expert judgment? with certainty it contains uncertainty," Journal of Loss Prevention in the Process Industries, vol. 66, Article ID 104200, 2020.

[26] X. K. Xu and S. Z. Hua, "Evaluation of government APP under the background of "internet + government service" based on intuitionistic fuzzy analytic hierarchy process," Journal of Information, vol. 39, no. 3, pp. 198-207, 2020.

[27] R. Şahin and P. Liu, "Correlation coefficient of single-valued neutrosophic hesitant fuzzy sets and its applications in decision making," Neural Computing \& Applications, vol. 28, no. 6, pp. 1387-1395, 2017.

[28] F. Teng and P. D. Liu, "A large group decision-making method based on a generalized shapley probabilistic linguistic choquet average operator and the TODIM method," Computers \& Industrial Engineering, vol. 151, Article ID 106971, 2021.

[29] D. F. Li, Intuitionistic Fuzzy Set Decision Making and Game Analysis, National Defense Industry Press, Beijing, China, 2012.

[30] K. T. Atanassov, "Intuitionistic fuzzy sets," Fuzzy Sets and Systems, vol. 20, pp. 87-96, 1986.

[31] R. R. Yager, "Pythagorean fuzzy subsets," in Proceedings of the 2013 Joint P1 IFSA World Congress and NAFIPS Annual Meeting, Edmonton, Canada, June 2013.

[32] X. Yager and Y. Yang, "Some results for pythagorean fuzzy sets," International Journal of Intelligent Systems, vol. 30, no. 11, pp. 1133-1160, 2015.

[33] P. Ren, Z. X, and X. Gou, "Pythagorean fuzzy TODIM approach to multi-criteria decision making," Applied Soft Computing, vol. 42, pp. 246-259, 2016.

[34] L. S. Shapley, A Value for n-Person Game, Princeton University Press, Princeton, NJ, USA, 1953.

[35] Y. Mu, J. Wu, N. Jenkins, H. Jia, and C. Wang, "A spatialtemporal model for grid impact analysis of plug-in electric vehicles," Applied Energy, vol. 114, pp. 456-465, 2014.

[36] X. Huang and J. Ge, "Electric vehicle development in Beijing: an analysis of consumer purchase intention," Journal of Cleaner Production, vol. 216, pp. 361-372, 2019.

[37] C. Csiszár, C. Bálint, D. Földes, E. Wirth, and L. Amás, "Location optimisation method for fast-charging stations along national roads," Journal of Transport Geography, vol. 88, Article ID 102833, 2020.

[38] Z. P. Liu, F. S. Wen, and Y. S. Xue, "Optimalsiting and sizing of distributed generators considering plug-inelectric vehicles," Automation of Electric Power Systems, vol. 35, no. 18, pp. 11-16, 2011.

[39] J. Wang, G.-W. Wei, C. Wei, and J. Wu, "Maximizing deviation method for multiple attribute decision making under q-rung orthopair fuzzy environment," Defence Technology, vol. 16, no. 5, pp. 1073-1087, 2020. 